\title{
Some common fixed-point and invariant approximation results with generalized almost contractions
}

Savita Rathee and Anil Kumar*

\section{"Correspondence:}

anilkshk84@gmail.com

Department of Mathematics,

Maharshi Dayanand University,

Rohtak, Haryana 124001, India

\begin{abstract}
In this paper, the concept of a generalized almost $(f, g)$-contraction is introduced and we establish some common fixed-point results for the noncommuting generalized almost $(f, g)$-contraction in the setup of metric spaces and normed linear spaces, where the set of fixed points of $f$ and $g$ need not be starshaped. As applications, invariant approximation results are proved. Supporting examples are also given.
\end{abstract}

Keywords: best approximation; Banach operator pair; generalized almost contraction; property $(\mathrm{N})$; jointly continuous contractive family

\section{Introduction}

The classical Banach contraction principle is a very popular tool for solving problems in nonlinear analysis. It has various applications to operator theory, variational analysis, and approximation theory, so it has been extended in many ways (see, e.g., [1-30]).

In 2004, Berinde [1] defined the notion of a weak contraction mapping, which is more general than a contraction mapping. However, in [2] Berinde renamed it as an almost contraction, which is more appropriate.

Definition 1.1 Let $(X, d)$ be a complete metric space. A map $T: X \rightarrow X$ is called an almost contraction if there exist a constant $\delta \in(0,1)$ and some $L \geq 0$ such that

$$
d(T x, T y) \leq \delta d(x, y)+L d(y, T x) \quad \text { for all } x, y \in X
$$

Berinde [1] proved some fixed-point theorems for almost contractions in a complete metric space which generalized the results of Kannan [3], Chatterjea [4], and Zamfirescu [5].

In 2008, Babu et al. [6] defined the class of mappings satisfying 'condition (B)' as follows.

Definition 1.2 Let $(X, d)$ be a metric space. A map $T: X \rightarrow X$ is said to satisfy 'condition (B)' if there exist a constant $\delta \in(0,1)$ and some $L \geq 0$ such that

$$
d(T x, T y) \leq \delta d(x, y)+L \min \{d(x, T x), d(y, T y), d(x, T y), d(y, T x)\}
$$

for all $x, y \in X$.

\section{Springer}

@2014 Rathee and Kumar: licensee Springer. This is an Open Access article distributed under the terms of the Creative Commons Attribution License (http://creativecommons.org/licenses/by/2.0), which permits unrestricted use, distribution, and reproduction in any medium, provided the original work is properly cited. 
They prove that any map $T$ satisfying 'condition (B)' has a unique fixed point in complete metric spaces. They also discuss quasi-contraction, almost contraction, and the class of mappings that satisfy 'condition (B)' in detail.

Afterwards Berinde [7] generalized the above definition and proved the following fixedpoint result.

Theorem 1.3 Let $(X, d)$ be a complete metric space and let $T: X \rightarrow X$ be a mapping for which there exist $\delta \in(0,1)$ and some $L \geq 0$ such that for all $x, y \in X$

$$
d(T x, T y) \leq \delta M_{1}(x, y)+L \min \{d(x, T x), d(y, T y), d(x, T y), d(y, T x)\},
$$

where

$$
M_{1}(x, y)=\max \left\{d(x, y), d(x, T x), d(y, T y), \frac{1}{2}[d(x, T y)+d(y, T x)]\right\} .
$$

Then T has a unique fixed point.

The contractive condition (1.3) is termed as generalized almost contraction.

Recently, Abbas and Ilić in [15] introduced the following definition.

Definition 1.4 Let $T$ and $f$ be two self-maps of a metric space $(X, d)$. A map $T$ is called a generalized almost $f$-contraction if there exist $\delta \in(0,1)$ and some $L \geq 0$ such that

$$
d(T x, T y) \leq \delta M_{1}(x, y)+L \min \{d(f x, T x), d(f y, T y), d(f x, T y), d(f y, T x)\}
$$

where

$$
M_{1}(x, y)=\max \left\{d(f x, f y), d(f x, T x), d(f y, T y), \frac{1}{2}[d(f x, T y)+d(f y, T x)]\right\} .
$$

If $f$ = identity map, then condition (1.3) can be obtained as particular case of condition (1.4). However, in [15] Abbas and Ilić obtained various common fixed-point and invariant approximation results for such mappings under the assumption of weak compatibility of maps.

Recently, Chen and Li [10] introduced the class of Banach operator pairs, as a new class of noncommuting mappings and obtained some common fixed-point and invariant approximation results for this class of maps. This class of noncommuting maps is different from the class of noncommuting maps ( $v i z$. $R$-subcommuting, $R$-sub-weakly commuting, $C_{q}$-commuting, compatible, weakly compatible etc.) studied in [11-13, 15, 17-19, 27-29]. So, it has been further studied by various authors (see, e.g., [16, 21, 22, 24]).

In this article, we introduce the class of generalized almost $(f, g)$-contraction and consequently establish some common fixed-point results for the noncommuting generalized almost $(f, g)$-contraction in the framework of metric spaces and normed linear spaces, where the set of fixed points of $f$ and $g$ need not be starshaped. As an application, invariant approximation results are proved. The proved results generalize and extend the corresponding results of Chen and Li [10], Al-Thagafi and Shahzad [16], Akbar et al. [22], Chandok and Narang [24], Al-Thagafi [25] and Jungck and Sessa [26], Shahzad [28] to the class of generalized almost $(f, g)$-contractions. 


\section{Preliminaries}

First, we introduce some well-known notations and definitions that will be needed in the sequel.

Let $(X, d)$ be a metric space, $M$ be a subset of $X$ and $f, T$ be self-maps of $M$. A point $x \in M$ is a coincidence point (common fixed point) of $f$ and $T$ if $f x=T x(f x=T x=x)$. The set of coincidence points of $f$ and $T$ is denoted by $C(f, T)$ and the set of fixed points of $f$ is denoted by $F(f)$. The pair $\{f, T\}$ is called

(1) commuting if $T f x=f T x$ for all $x \in M$,

(2) compatible [8] if $\lim _{n \rightarrow \infty} d\left(T f x_{n}, f T x_{n}\right)=0$ whenever $\left\{x_{n}\right\}$ is a sequence in $M$ such that $\lim _{n \rightarrow \infty} f x_{n}=\lim _{n \rightarrow \infty} T x_{n}=t$ for some $t \in M$,

(3) weakly compatible [9] if $T f x=f T x$ for all $x \in C(f, T)$,

(4) a Banach operator pair [10] if the set $F(f)$ is $T$-invariant, namely $T(F(f)) \subseteq F(f)$.

Obviously, a commuting pair $(T, f)$ is a Banach operator pair but not conversely. If $(T, f)$ is a Banach operator pair, then $(f, T)$ need not be Banach operator pair (see [10]).

Let $M$ be a subset of a normed space $(X,\|\cdot\|)$. The set $B_{M}(p)=\{x \in M:\|x-p\|=$ $\operatorname{dist}(p, M)\}$ is called the set of best approximants to $p \in X$ out of $M$, where $\operatorname{dist}(p, M)=$ $\inf \{\|y-p\|: p \in M\}$. We denote by $\mathbb{N}$ and $\operatorname{cl}(M)(\operatorname{wcl}(M))$ the set of positive integers and the closure (weak closure) of a set $M$ in $X$, respectively.

The set $M$ is said to be (a) $q$-starshaped if there exists $q \in M$ such that the line segment $[q, x]=\{(1-k) q+k x: 0 \leq k \leq 1\}$ joining $q$ to $x$ is contained in $M$ for all $x \in M$; (b) convex if $k x+(1-k) y \in M$ for all $x, y \in M$. The map $f$ defined on a set $M$ is called

(1) affine [11] if $M$ is convex and $f((1-k) y+k x)=(1-k) f y+k f x$, for all $x, y \in M$,

(2) $q$-affine [11] if $M$ is $q$-starshaped and $f((1-k) q+k x)=(1-k) q+k f x$, for all $x \in M$.

Suppose that $M$ is $q$-starshaped with $q \in F(f)$ and is both $T$ - and $f$-invariant. Then $T$ and $f$ are called

(1) $C_{q}$-commuting [11] if $f T x=T f x$ for all $x \in C_{q}(f, T)$, where $C_{q}(f, T)=\bigcup\left\{C\left(f, T_{k}\right): 0 \leq k \leq 1\right\}$ where $T_{k}(x)=(1-k) q+k T x$,

(2) $R$-subcommuting on $M$ [12] if, for all $x \in M$, there exists a real number $R>0$ such that $\|T f x-f T x\| \leq \frac{R}{k}\|k T x+(1-k) q-f x\|, 0<k \leq 1$,

(3) $R$-sub-weakly commuting on $M$ [13] if, for all $x \in M$, there exists a real number $R>0$ such that $\|T f x-f T x\| \leq R \operatorname{dist}(f x,[q, T x])$.

A Banach space $X$ is said to satisfy Opial's condition if, whenever $\left\{x_{n}\right\}$ is a sequence in $X$ such that $\left\{x_{n}\right\}$ converges weakly to $x \in X$, the inequality

$$
\liminf _{n \rightarrow \infty}\left\|x_{n}-x\right\|<\liminf _{n \rightarrow \infty}\left\|x_{n}-y\right\|
$$

holds for all $y \neq x$. A Hilbert space and the space $l_{p}(1<p<\infty)$ satisfy Opial's condition. The map $T: M \rightarrow X$ is said to be demiclosed at zero if, whenever $\left\{x_{n}\right\}$ is a sequence in $M$ such that $\left\{x_{n}\right\}$ converges weakly to $x \in M$ and $\left\{T x_{n}\right\}$ converges to 0 , then $T x=0$.

The following important extension of the concept of starshapedness was defined by Naimpally et al. [14] and has been studied by many authors.

Definition 2.1 A subset $M$ of a linear space $X$ is said to have property $(\mathrm{N})$ with respect to $T$ if

(1) $T: M \rightarrow M$,

(2) $\left(1-k_{n}\right) q+k_{n} T x \in M$, for some $q \in M$ and a fixed sequence of real numbers $k_{n}$ $\left(0<k_{n}<1\right)$ converging to 1 and for each $x \in M$. 
It is to be noted that each $T$-invariant $q$-starshaped set has property $(\mathrm{N})$ but converse does not hold in general. This is shown by the following example.

Example 2.2 Let $X=\mathfrak{R}$ be the set of real numbers and $M=\{1 / n$, where $n$ is a natural number be endowed with the usual norm. Define $T x=1$ for each $x \in M$. Then clearly $M$ is not $q$-starshaped but has property (N) with respect to $T$, for $q=1, k_{n}=1-1 / n$.

\section{Main results}

First we introduce the notion of a generalized almost $(f, g)$-contraction.

Definition 3.1 Let $(X, d)$ be a metric space and $f, g$ be self-maps of $X$. A mapping $T: X \rightarrow$ $X$ is said to be a generalized almost $(f, g)$-contraction if there exist $\delta \in(0,1)$ and some $L \geq 0$ such that

$$
d(T x, T y) \leq \delta M_{1}(x, y)+L N_{1}(x, y) \quad \text { for all } x, y \in X
$$

where

$$
M_{1}(x, y)=\max \left\{d(f x, g y), d(f x, T x), d(g y, T y), \frac{1}{2}[d(f x, T y)+d(g y, T x)]\right\}
$$

and

$$
N_{1}(x, y)=\min \{d(f x, T x), d(g y, T y), d(f x, T y), d(g y, T x)\} .
$$

If $g=f$, then Definition 1.4 is a particular case of Definition 3.1. If $g=f=I$ (identity operator), then equation (1.3) can be obtained as a special case of equation (3.1).

Here we observe that if $T$ satisfies 'condition (B)' then $T$ is a generalized almost contraction but its converse need not be true. This is shown by the following example.

Example 3.2 Let $X=[0, \infty)$ be endowed with the Euclidean metric $d(x, y)=|x-y|$. We define a mapping $T: X \rightarrow X$ by

$$
T(x)= \begin{cases}\frac{3}{4} & \text { if } 0 \leq x \leq 1 \\ \frac{1}{2} & \text { if } 0 \leq x<\infty\end{cases}
$$

Then $T$ is a generalized almost contraction with $\delta=\frac{2}{3}$ and $L=0$. But $T$ does not satisfy 'condition (B)' at $x=\frac{3}{4}, y=1$ for any $\delta \in(0,1)$ and $L \geq 0$.

In (3.1) if $L=0$, then $T$ is called a generalized $(f, g)$-contraction. Obviously, a generalized $(f, g)$-contraction implies a generalized almost $(f, g)$-contraction, but the converse is not true in general.

Example 3.3 Let $X=\{0,1,2\}$ with the usual metric and $f, g: X \rightarrow X$ be given by $f(x)=$ $g(x)=1$ for all $x \in X$. Also define a mapping $T: X \rightarrow X$ as

$$
T(x)= \begin{cases}0, & x \in\{0,2\} \\ 2, & x=1\end{cases}
$$


Then $T$ is a generalized almost $(f, g)$-contraction with any $\delta \in(0,1)$ and $L \geq 2$. But $T$ is not a generalized $(f, g)$-contraction at $x=0, y=1$ or $x=1, y=2$ for any $\delta \in(0,1)$.

The following lemma is a particular case of the main theorem of Abbas and Ilić [15].

Lemma 3.4 Let $M$ be a nonempty subset of a metric space $(X, d)$, and $T$ be a self-map of $M$. Assume that $\mathrm{cl}(T(M)) \subseteq M, \operatorname{cl}(T(M))$ is complete, and $T$ is a generalized almost contraction. Then $M \cap F(T)$ is singleton.

Now, we start with the following common fixed-point result, which will be used in sequel.

Theorem 3.5 Let $M$ be a nonempty subset of a metric space $(X, d)$, and $T, f$ and $g$ be selfmaps of $M$. Assume that $F(f) \cap F(g)$ is nonempty, $\operatorname{cl}(T(F(f) \cap F(g))) \subseteq F(f) \cap F(g), \operatorname{cl}(T(M))$ is complete, and $T$ is a generalized almost $(f, g)$-contraction. Then $M \cap F(T) \cap F(f) \cap F(g)$ is singleton.

Proof The completeness of $\operatorname{cl}(T(M))$ implies that of $\operatorname{cl}(T(F(f) \cap F(g)))$. Further, by a generalized almost $(f, g)$-contraction of $T$, for all $x, y \in F(f) \cap F(g)$, we have

$$
\begin{aligned}
d(T x, T y) \leq & \delta M_{1}(x, y)+L N_{1}(x, y) \\
= & \delta \max \left\{d(x, y), d(x, T x), d(y, T y), \frac{1}{2}[d(x, T y)+d(y, T x)]\right\} \\
& +L \min \{d(x, T x), d(y, T y), d(x, T y), d(y, T x)\} .
\end{aligned}
$$

Hence $T$ is a generalized almost contraction mapping on $F(f) \cap F(g)$ and $\operatorname{cl}(T(F(f) \cap$ $F(g))) \subseteq F(f) \cap F(g)$. By Lemma 3.4, $T$ has a unique fixed point $z$ in $F(f) \cap F(g)$ and consequently $M \cap F(T) \cap F(f) \cap F(g)$ is singleton.

Corollary 3.6 Let $M$ be a nonempty subset of a metric space $(X, d)$, and $T, f$ and $g$ be self-maps of $M$ such that $(T, f)$ and $(T, g)$ are Banach operator pairs on $M$. Assume that $\mathrm{cl}(T(M))$ is complete, $T$ is a generalized almost $(f, g)$-contraction and $F(f) \cap F(g)$ is nonempty and closed. Then $M \cap F(T) \cap F(f) \cap F(g)$ is singleton.

In Theorem 3.5 if we take $L=0$, then we easily obtain the following result, which improves and extends Lemma 3.1 of Chen and Li [10] and Theorem 2.2 of Al-Thagafi and Shahzad [16].

Corollary 3.7 Let $M$ be a nonempty subset of a metric space $(X, d)$, and $T, f$, and $g$ be self-maps on $M$. Assume that $F(f) \cap F(g)$ is nonempty, $\operatorname{cl}(T(F(f) \cap F(g))) \subseteq F(f) \cap F(g)$, $\operatorname{cl}(T(M))$ is complete, and $T$ is a generalized $(f, g)$-contraction. Then $M \cap F(T) \cap F(f) \cap F(g)$ is singleton.

Remark 3.8 By comparing Theorem 2.1 of Shahzad [17] with Corollary 3.7 (when $g=f$ ), their assumptions that $M$ is closed, $T(M) \subseteq f(M), T$ is continuous and $(T, f)$ is $R$-weakly commuting pair on $M$ are replaced with ' $F(f)$ is nonempty, $\operatorname{cl}(T(F(f))) \subseteq F(f)$ '. 
Theorem 3.9 Let $M$ be a nonempty subset of a normed (respectively, Banach) space $X$ and $T, f$, and $g$ be self-maps of $M$. If $F(f) \cap F(g)$ has the property $(\mathrm{N})$ with respect to $T$, $\operatorname{cl}(T(F(f) \cap F(g))) \subseteq F(f) \cap F(g)$ (respectively, $\operatorname{wcl}(T(F(f) \cap F(g))) \subseteq F(f) \cap F(g))$, and there exists a constant $L \geq 0$ such that

$$
\|T x-T y\| \leq m(x, y)+\operatorname{Ln}(x, y) \quad \text { for all } x, y \in M
$$

where

$$
\begin{aligned}
m(x, y)= & \max \{\|f x-g y\|, \operatorname{dist}(f x,[q, T x]), \operatorname{dist}(g y,[q, T y]), \\
& \left.\frac{1}{2}[\operatorname{dist}(g y,[q, T x])+\operatorname{dist}(f x,[q, T y])]\right\}
\end{aligned}
$$

and

$$
n(x, y)=\min \{\operatorname{dist}(f x,[q, T x]), \operatorname{dist}(g y,[q, T y]), \operatorname{dist}(g y,[q, T x]), \operatorname{dist}(f x,[q, T y])\}
$$

then $M \cap F(T) \cap F(f) \cap F(g) \neq \phi$, provided $\mathrm{cl}(T(M))$ is compact (respectively, $\mathrm{wcl}(T(M))$ is weakly compact) and $T$ is continuous (respectively, $I-T$ is demiclosed at 0 , where I stands for identity map).

Proof As $T(F(f) \cap F(g)) \subseteq F(f) \cap F(g)$ and $F(f) \cap F(g)$ has the property (N) with respect to $T$, for each $n \in \mathbb{N}$, we can define $T_{n}: F(f) \cap F(g) \rightarrow F(f) \cap F(g)$ by $T_{n} x=\left(1-k_{n}\right) q+k_{n} T x$ for all $x \in F(f) \cap F(g)$ and a fixed sequence of real numbers $k_{n}\left(0<k_{n}<1\right)$ converging to 1 . Since $F(f) \cap F(g)$ has the property (N) with respect to $T$, and $\operatorname{cl}(T(F(f) \cap F(g))) \subseteq F(f) \cap F(g)$ (respectively, wcl $(T(F(f) \cap F(g))) \subseteq F(f) \cap F(g))$, we have $\operatorname{cl}\left(T_{n}(F(f) \cap F(g))\right) \subseteq F(f) \cap F(g)$ (respectively, $\left.\operatorname{wcl}\left(T_{n}(F(f) \cap F(g))\right) \subseteq F(f) \cap F(g)\right)$ for each $n \in \mathbb{N}$. Also, by the inequality (3.2),

$$
\begin{aligned}
\left\|T_{n} x-T_{n} y\right\| & =k_{n}\|T x-T y\| \\
& \leq k_{n}[m(x, y)+\operatorname{Ln}(x, y)] \\
& =k_{n} m(x, y)+L_{n} n(x, y),
\end{aligned}
$$

where

$$
\begin{aligned}
m(x, y)= & \max \{\|f x-g y\|, \operatorname{dist}(f x,[q, T x]), \operatorname{dist}(g y,[q, T y]), \\
& \left.\frac{1}{2}[\operatorname{dist}(g y,[q, T x])+\operatorname{dist}(f x,[q, T y])]\right\} \\
\leq & \max \left\{\|f x-g y\|,\left\|f x-T_{n} x\right\|,\left\|g y-T_{n} y\right\|,\right. \\
& \left.\frac{1}{2}\left[\left\|f x-T_{n} y\right\|+\left\|g y-T_{n} x\right\|\right]\right\}
\end{aligned}
$$

and

$$
\begin{aligned}
n(x, y) & =\min \{\operatorname{dist}(f x,[q, T x]), \operatorname{dist}(g y,[q, T y]), \operatorname{dist}(g y,[q, T x]), \operatorname{dist}(f x,[q, T y])\} \\
& \leq \min \left\{\left\|f x-T_{n} x\right\|,\left\|g y-T_{n} y\right\|,\left\|f x-T_{n} y\right\|,\left\|g y-T_{n} x\right\|\right\}
\end{aligned}
$$


for all $x, y \in F(f) \cap F(g), L_{n}:=k_{n} L$, and $0<k_{n}<1$. Thus, for each $n \in \mathbb{N}, T_{n}$ is a generalized $(f, g)$-almost contraction.

If $\operatorname{cl}(T(M))$ is compact, then, for each $n \in \mathbb{N}, \operatorname{cl}\left(T_{n}(M)\right)$ is compact and hence complete. By Theorem 3.5, for each $n \geq 1$, there is a unique $x_{n}$ in $M$ such that $x_{n}=f\left(x_{n}\right)=g\left(x_{n}\right)=$ $T_{n}\left(x_{n}\right)$. The compactness of $\operatorname{cl}(T(M))$ implies that there exists a subsequence $\left\{T x_{m}\right\}$ of $\left\{T x_{n}\right\}$ such that $T x_{m} \rightarrow z \in \operatorname{cl}(T(M))$. Since $\left\{T x_{m}\right\}$ is a sequence in $T(F(f) \cap F(g))$ and $\operatorname{cl}(T(F(f) \cap F(g))) \subseteq F(f) \cap F(g)$, we have $z \in F(f) \cap F(g)$. Moreover,

$$
x_{m}=T_{m}\left(x_{m}\right)=\left(1-k_{m}\right) q+k_{m} T x_{m} \rightarrow z .
$$

As $T$ is continuous on $M$, we have $T z=z$. Thus $M \cap F(T) \cap F(f) \cap F(g) \neq \phi$.

Next, the weak compactness of $\operatorname{wcl}(T(M))$ implies that $\operatorname{wcl}\left(T_{n}(M)\right)$ is weakly compact and hence complete due to completeness of $X$. From Theorem 3.5, for each $n \geq 1$, there is a unique $x_{n}$ in $M$ such that $x_{n}=f\left(x_{n}\right)=g\left(x_{n}\right)=T_{n}\left(x_{n}\right)$. The weak compactness of $\mathrm{wcl}(T(M))$ implies that there is a subsequence $\left\{T x_{m}\right\}$ of $\left\{T x_{n}\right\}$ such that $T x_{m}$ converges weakly to $z \in \operatorname{wcl}(T(M))$. Since $\left\{T x_{m}\right\}$ is a sequence in $T(F(f) \cap F(g))$ and $\operatorname{wcl}(T(F(f) \cap F(g))) \subseteq$ $F(f) \cap F(g)$, therefore $z \in F(f) \cap F(g)$. Also we have $(I-T) x_{m} \rightarrow 0$ as $m \rightarrow \infty$. Further, demiclosedness of $I-T$ at 0 implies $z=T z$, thus $M \cap F(T) \cap F(f) \cap F(g) \neq \phi$.

Corollary 3.10 Let $M$ be a nonempty subset of a normed (respectively, Banach) space $X$ and $T, f$, and $g$ be self-maps of $M$. If $F(f) \cap F(g)$ has the property $(\mathrm{N})$ with respect to $T$ and is closed (respectively, weakly closed), $(T, f)$ and $(T, g)$ are Banach operator pairs and satisfy (3.2) for all $x, y \in M$. Then $M \cap F(T) \cap F(f) \cap F(g) \neq \phi$, provided $\mathrm{cl}(T(M))$ is compact (respectively, $\operatorname{wcl}(T(M))$ is weakly compact) and $T$ is continuous (respectively, $I-T$ is demiclosed at 0 , where I stands for the identity map).

Remark 3.11 (1) By comparing Theorem 2.2 of Shahzad [17] with the first case of Theorem 3.9 (when $g=f, L=0$ ), their assumptions ' $q \in F(f), M$ is closed and $q$-starshaped, $f$ is linear and continuous on $M, T(M) \subseteq f(M)$ and $(T, f)$ is $R$-sub-weakly commuting pair on $M$ ' are replaced with ' $M$ is a nonempty subset, $F(f)$ has the property $(\mathrm{N})$ with respect to $T, \mathrm{cl}(T(F(f))) \subseteq F(f)$ '.

(2) By comparing Theorem 2.2(i) of Hussain and Jungck [18] with the first case of Theorem 3.9 (when $L=0$ ), their assumptions ' $M$ is complete and $q$-starshaped, $f$ and $g$ are continuous and affine on $M, T(M) \subseteq f(M) \cap g(M), q \in F(f) \cap F(g)$, and $(T, f)$ and $(T, g)$ are $R$-sub-weakly commuting pair on $M$ ' are replaced with ' $F(f) \cap F(g)$ has the property (N) with respect to $T, \operatorname{cl}(T(F(f) \cap F(g))) \subseteq F(f) \cap F(g)$ '.

(3) By comparing Theorem 2.2(ii) of Hussain and Jungck [18] with the second case of Theorem 3.9 (when $L=0$ ), their assumptions ' $M$ is weakly compact and $q$-starshaped, $f$ and $g$ are affine and continuous on $M, T(M) \subseteq f(M) \cap g(M), q \in F(f) \cap F(g)$, and $(T, f)$ and $(T, g)$ are $R$-sub-weakly commuting pair on $M$, and $f-T$ is demiclosed at 0 ' are replaced with 'wcl $(T(M))$ is weakly compact, $F(f) \cap F(g)$ has the property $(\mathrm{N})$ with respect to $T$, $\operatorname{wcl}(T(F(f) \cap F(g))) \subseteq F(f) \cap F(g)$ and $I-T$ is demiclosed at 0 '.

Remark 3.12 If the contractive condition (3.2) in Theorem 3.9 is replaced with the stronger contractive condition

$$
\|T x-T y\| \leq m(x, y)+\operatorname{Ln}(x, y)
$$


for all $x, y \in M$ and some $L \geq 0$, where

$$
\begin{aligned}
m(x, y)= & \max \left\{\|f x-g y\|, \frac{1}{2}[\operatorname{dist}(f x,[q, T x])+\operatorname{dist}(g y,[q, T y])],\right. \\
& \left.\frac{1}{2}[\operatorname{dist}(g y,[q, T x])+\operatorname{dist}(f x,[q, T y])]\right\}
\end{aligned}
$$

and

$$
n(x, y)=\min \{\operatorname{dist}(f x,[q, T x]), \operatorname{dist}(g y,[q, T y]), \operatorname{dist}(g y,[q, T x]), \operatorname{dist}(f x,[q, T y])\},
$$

then continuity of $T$ can be relaxed in the first case of Theorem 3.9.

Proof The proof will be similar to the first case of Theorem 3.9. To prove $T z=z$, instead of continuity of $T$, using (3.3) we have

$$
\left\|T x_{m}-T z\right\| \leq m\left(x_{m}, z\right)+\operatorname{Ln}\left(x_{m}, z\right)
$$

where

$$
\begin{aligned}
m\left(x_{m}, z\right)= & \max \left\{\left\|f x_{m}-g z\right\|, \frac{1}{2}\left[\operatorname{dist}\left(f x_{m},\left[q, T x_{m}\right]\right)+\operatorname{dist}(g z,[q, T z])\right],\right. \\
& \left.\frac{1}{2}\left[\operatorname{dist}\left(g z,\left[q, T x_{m}\right]\right)+\operatorname{dist}\left(f x_{m},[q, T z]\right)\right]\right\} \\
\leq & \max \left\{\left\|x_{m}-z\right\|, \frac{1}{2}\left[\left\|x_{m}-T x_{m}\right\|+\|z-T z\|\right],\right. \\
& \left.\frac{1}{2}\left[\left\|z-T x_{m}\right\|+\left\|x_{m}-T z\right\|\right]\right\} \\
\leq & \max \left\{\left\|x_{m}-z\right\|, \frac{1}{2}\left[\left\|x_{m}-T x_{m}\right\|+\left\|z-T x_{m}\right\|+\left\|T x_{m}-T z\right\|\right],\right. \\
& \left.\frac{1}{2}\left[\left\|z-T x_{m}\right\|+\left\|x_{m}-T x_{m}\right\|+\left\|T x_{m}-T z\right\|\right]\right\}
\end{aligned}
$$

and

$$
\begin{aligned}
n\left(x_{m}, z\right)= & \min \left\{\operatorname{dist}\left(f x_{m},\left[q, T x_{m}\right]\right), \operatorname{dist}(g z,[q, T z]),\right. \\
& \left.\operatorname{dist}\left(g z,\left[q, T x_{m}\right]\right), \operatorname{dist}\left(f x_{m},[q, T z]\right)\right\} \\
\leq & \min \left\{\left\|x_{m}-T x_{m}\right\|,\|z-T z\|,\left\|z-T x_{m}\right\|,\left\|x_{m}-T z\right\|\right\} .
\end{aligned}
$$

Now taking $m \rightarrow \infty$ in (3.4) we can write

$$
\lim _{m \rightarrow \infty}\left\|T x_{m}-T z\right\| \leq \frac{1}{2} \lim _{m \rightarrow \infty}\left\|T x_{m}-T z\right\|
$$

This is possible only if $T x_{m} \rightarrow T z$ as $m \rightarrow \infty$, which implies $T z=z$.

Let $C=B_{M}(p) \cap C_{M}^{f, g}(p)$, where $C_{M}^{f, g}(p)=\left\{x \in M: f x \in B_{M}(p), g x \in B_{M}(p)\right\}$. 
Corollary 3.13 Let $X$ be a normed (respectively, Banach) space and let $T, f$, and $g$ be selfmaps of $X$. If $p \in X$ and $D \subseteq C, D_{0}:=D \cap F(f) \cap F(g)$ has the property $(\mathrm{N})$ with respect to $T, \mathrm{cl}\left(T\left(D_{0}\right)\right) \subseteq D_{0}$ (respectively, $\left.\operatorname{wcl}\left(T\left(D_{0}\right)\right) \subseteq D_{0}\right), \operatorname{cl}(T(D))$ is compact (respectively, wcl $(T(D))$ is weakly compact), $T$ is continuous on $D$ (respectively, $I-T$ is demiclosed at 0 , where I stands for identity map) and (3.2) holds for all $x, y \in D$, then $B_{M}(p) \cap F(T) \cap F(f) \cap$ $F(g) \neq \phi$.

Corollary 3.14 Let $X$ be a normed (respectively, Banach) space and let $T, f$, and $g$ be selfmaps of $X$. If $p \in X$ and $D \subseteq B_{M}(p), D_{0}:=D \cap F(f) \cap F(g)$ has the property $(\mathrm{N})$ with respect to $T, \operatorname{cl}\left(T\left(D_{0}\right)\right) \subseteq D_{0}$ (respectively,, $\left.\mathrm{wcl}\left(T\left(D_{0}\right)\right) \subseteq D_{0}\right), \operatorname{cl}(T(D))$ is compact (respectively, $\operatorname{wcl}(T(D))$ is weakly compact), $T$ is continuous on $D$ (respectively, $I-T$ is demiclosed at 0 , where I stands for the identity map) and (3.2) holds for all $x, y \in D$, then $B_{M}(p) \cap F(T) \cap$ $F(f) \cap F(g) \neq \phi$.

Remark 3.15 Corollaries 3.13 and 3.14 improve and develop Theorems 2.8-2.11 of Hussain and Jungck [18] and Theorems 3.1-3.4 of Song [19] to the non-starshaped domain.

Denote by $\mathcal{L}_{0}$ the class of closed convex subsets of $X$ containing 0 . For $M \in \mathcal{L}_{0}$, we define $M_{p}=\{x \in M:\|x\| \leq 2\|p\|\}$. Clearly $B_{M}(p) \subseteq M_{p} \in \mathcal{L}_{0}$.

The following invariant approximation result constitutes an extension of Theorem 2.6 of Al-Thagafi and Shahzad [16] and Corollary 2.10 of [29] to a non-starshaped domain.

Theorem 3.16 Let $X$ be a normed (respectively, Banach) space and $T, f, g: X \rightarrow X$. If $p \in$ $X$ and $M \in \mathcal{L}_{0}$ such that $T\left(M_{p}\right) \subseteq M, \operatorname{cl}\left(T\left(M_{p}\right)\right)$ is compact (respectively, $\operatorname{wcl}\left(T\left(M_{p}\right)\right)$ is weakly compact), and $\|T x-p\| \leq\|x-p\|$ for all $x \in M_{p}$, then $B_{M}(p)$ is nonempty, closed, and convex with $T\left(B_{M}(p)\right) \subseteq B_{M}(p)$. If, in addition, $D$ is a subset of $B_{M}(p), D_{0}:=D \cap F(f) \cap F(g)$ has the property $(\mathrm{N})$ with respect to $T, \operatorname{cl}\left(T\left(D_{0}\right)\right) \subseteq D_{0}$ (respectively, $\left.\operatorname{wcl}\left(T\left(D_{0}\right)\right) \subseteq D_{0}\right), T$ is continuous on $D$ (respectively, $I-T$ is demiclosed at 0 , where $I$ stands for the identity map) and (3.2) holds for all $x, y \in D$, then $B_{M}(p) \cap F(T) \cap F(f) \cap F(g) \neq \phi$.

Proof We may assume that $p \notin M$. If $y \in M \backslash M_{p}$, then $\|y\|>2\|p\|$ and, so

$$
\|y-p\| \geq\|y\|-\|p\|>\|p\| \geq \operatorname{dist}(p, M) .
$$

Thus $\operatorname{dist}\left(p, M_{p}\right)=\operatorname{dist}(p, M)$. Assume that $\operatorname{cl}\left(T\left(M_{p}\right)\right)$ is compact, then by the continuity of the norm there exists $z \in \operatorname{cl}\left(T\left(M_{p}\right)\right)$ such that $\|z-p\|=\operatorname{dist}\left(p, c l T\left(M_{p}\right)\right)$.

If we assume that $\mathrm{wcl}\left(T\left(M_{p}\right)\right)$ is weakly compact, then by using Lemma 5.5 of [20, p.192] we can show the existence of $z \in \operatorname{wcl}\left(T\left(M_{p}\right)\right)$ such that $\|z-p\|=\operatorname{dist}\left(p, \operatorname{wcl} T\left(M_{p}\right)\right)$. Thus in both cases, we have

$$
\operatorname{dist}\left(p, M_{p}\right) \leq \operatorname{dist}\left(p, \operatorname{cl} T\left(M_{p}\right)\right) \leq \operatorname{dist}\left(p, T\left(M_{p}\right)\right) \leq\|T x-p\| \leq\|x-p\|
$$

for all $x \in M_{p}$. It follows that $\|z-p\|=\operatorname{dist}(p, M)$. Thus $B_{M}(p)$ is nonempty, closed, and convex with $T\left(B_{M}(p)\right) \subseteq B_{M}(p)$. The compactness of $\operatorname{cl}\left(T\left(M_{p}\right)\right)$ (respectively, weak compactness of $\left.\operatorname{wcl}\left(T\left(M_{p}\right)\right)\right)$ implies that $\operatorname{cl}(T(D))$ is compact (respectively, $\operatorname{wcl}(T(D))$ is weakly compact). Then by Corollary 3.14, $B_{M}(p) \cap F(T) \cap F(f) \cap F(g) \neq \phi$. 
Now, we present some non-trivial examples in support of Theorem 3.9.

Example 3.17 Let $X=\mathfrak{R}$ be the set of real numbers with the usual norm and $M=[0,1)$. We define mappings $f, g, T: M \rightarrow M$ by

$$
f(x)=\left\{\begin{array}{ll}
0 & \text { if } 0 \leq x<\frac{2}{3}, \\
\frac{4}{3}-x & \text { if } \frac{2}{3} \leq x<1,
\end{array} \quad g(x)= \begin{cases}\frac{x}{2} & \text { if } 0 \leq x<\frac{2}{3} \\
\frac{2}{3} & \text { if } \frac{2}{3} \leq x<1\end{cases}\right.
$$

and $T(x)=\frac{2}{3}$, for $0 \leq x<1$.

Here we observe that $F(f) \cap F(g)=\left\{0, \frac{2}{3}\right\}, \operatorname{cl}(T(F(f) \cap F(g)))=\left\{\frac{2}{3}\right\} \subseteq F(f) \cap F(g)$ and $\operatorname{cl}(T(M))=\left\{\frac{2}{3}\right\}$ is compact. Clearly $F(f) \cap F(g)$ is not starshaped but has property (N) with respect to $\mathrm{T}$, for $q=\frac{2}{3}$ and $k_{n}=1-1 / n$. Further, the mappings $T, f$, and $g$ satisfy the contractive condition (3.2) and also $T$ is continuous. Hence all the conditions of the first case of Theorem 3.9 are satisfied and consequently $T, f$, and $g$ have a common fixed point, $x=\frac{2}{3}$.

Remark 3.18 In Example 3.17, it is interesting to note that Theorem 2.19 of Hussain and Cho [21], and Corollary 3.10 of Akbar et al. [22] cannot apply, since $F(f) \cap F(g)$ is not $q$-starshaped.

Example 3.19 Let $X=\Re$ be the set of real numbers with the usual norm and $M=[0,1]$. Define $f, g, T: M \rightarrow M$ by

$$
f(x)=\left\{\begin{array}{ll}
x, & x \text { is rational in } M, \\
0, & \text { otherwise, }
\end{array} \quad g(x)=x \quad \text { for all } x \in M\right.
$$

and

$$
T(x)= \begin{cases}1 & \text { if } 0 \leq x<1 \\ 0 & \text { if } x=1\end{cases}
$$

Clearly $F(f) \cap F(g)=\{x, x$ is rational in $M\}$ has property $(\mathrm{N})$ with respect to $T$, for $q=0$, $k_{n}=1-1 / n$. Further, $\operatorname{cl}(T(F(f) \cap F(g)))=\{0,1\} \subseteq F(f) \cap F(g), \operatorname{cl}(T(M))=\{0,1\}$ is compact and $T, f$, and $g$ satisfy the contractive condition (3.2). Hence all the conditions of the first case of Theorem 3.9 are satisfied except the continuity of $T$. Note that $F(T) \cap F(f) \cap$ $F(g)=\phi$.

Remark 3.20 It is to be noted that the maps $T$, $f$, and $g$ given in Example 3.19 do not satisfy the contractive condition (3.3) at the point $x=\frac{1}{2}, y=1$.

\section{Results with joint contractive family}

Dotson [23] proved some results concerning the existence of fixed points of nonexpansive mappings on a certain class of non-convex sets. For proving these results, he extends the concept of starshapedness by introducing the following class of non-convex set.

Let $M$ be a subset of a normed space $X$ and $\Gamma=\left\{h_{x}: x \in M\right\}$ be a family of functions from $[0,1]$ to $M$ such that $h_{x}(1)=x$ for each $x \in M$. The family $\Gamma$ is said to be contractive if there exists a function $\varphi:(0,1) \rightarrow(0,1)$ such that for all $x, y \in M$ and all $t \in(0,1)$, we have

$$
\left\|h_{x}(t)-h_{y}(t)\right\| \leq \varphi(t)\|x-y\| .
$$


Such a family $\Gamma$ is said to be jointly continuous (jointly weakly continuous) if $t \rightarrow t_{0}$ in $[0,1]$ and $x \rightarrow x_{0}\left(x \rightarrow x_{0}\right.$ weakly) in $M$; then $h_{x}(t) \rightarrow h_{x_{0}}(t)\left(h_{x}(t) \rightarrow h_{x_{0}}(t)\right.$ weakly) in $M$.

We observe that if $M$ is $q$-starshaped subset of a normed linear space $X$ and $h_{x}(t)=$ $(1-t) q+t x$, for each $x \in M, q \in M$ and $t \in[0,1]$, then $\Gamma$ is a contractive jointly continuous and jointly weakly continuous family with $\varphi(t)=t$. Thus the class of subsets of $X$ with the property of contractiveness and joint continuity contains the class of starshaped sets which in turns contains the class of convex sets.

We shall denote $Y_{q}^{T x}=\left\{h_{T x}(k): 0 \leq k \leq 1\right\}$ where $q=h_{T x}(0)$.

The following results properly contain Theorems 3.2 and 3.3 of [10], Theorems 1 and 2 of [24] and improves Theorem 2.2 of [25], Theorem 6 of [26].

Theorem 4.1 Let $M$ be a nonempty subset of a normed (respectively, Banach) space $X$ and $T, f$ and $g$ be self-maps of $M$. Suppose $F(f) \cap F(g)$ is nonempty and has a contractive, jointly continuous (respectively, jointly weakly continuous) family of functions $\Gamma=\left\{h_{x}: x \in F(f) \cap\right.$ $F(g)\}, \operatorname{cl}(T(F(f) \cap F(g))) \subseteq F(f) \cap F(g)$ (respectively, $\operatorname{wcl}(T(F(f) \cap F(g))) \subseteq F(f) \cap F(g))$, and there exists a constant $L \geq 0$ such that

$$
\|T x-T y\| \leq m(x, y)+\operatorname{Ln}(x, y)
$$

for all $x, y \in M$, where

$$
m(x, y)=\max \left\{\|f x-g y\|, \operatorname{dist}\left(f x, Y_{q}^{T x}\right), \operatorname{dist}\left(g y, Y_{q}^{T y}\right), \frac{1}{2}\left[\operatorname{dist}\left(g y, Y_{q}^{T x}\right)+\operatorname{dist}\left(f x, Y_{q}^{T y}\right)\right]\right\}
$$

and

$$
n(x, y)=\min \left\{\operatorname{dist}\left(f x, Y_{q}^{T x}\right), \operatorname{dist}\left(g y, Y_{q}^{T y}\right), \operatorname{dist}\left(g y, Y_{q}^{T x}\right), \operatorname{dist}\left(f x, Y_{q}^{T y}\right)\right\}
$$

Then $M \cap F(T) \cap F(f) \cap F(g) \neq \phi$, provided $\mathrm{cl}(T(M))$ is compact (respectively, $\operatorname{wcl}(T(M)$ ) is weakly compact) and $T$ is continuous (respectively, $T$ is weakly continuous).

Proof For each natural number $n$, let $k_{n}=\frac{n}{n+1}$. Define $T_{n}: F(f) \cap F(g) \rightarrow F(f) \cap F(g)$ by $T_{n}(x)=h_{T x}\left(k_{n}\right)$ for all $x \in F(f) \cap F(g)$. Since $F(f) \cap F(g)$ has a contractive family and $\operatorname{cl}(T(F(f) \cap F(g))) \subseteq F(f) \cap F(g)$ (respectively, wcl $(T(F(f) \cap F(g))) \subseteq F(f) \cap F(g))$, so for each $n \in \mathbb{N}, \operatorname{cl}\left(T_{n}(F(f) \cap F(g))\right) \subseteq F(f) \cap F(g)$ (respectively, $\left.\operatorname{wcl}\left(T_{n}(F(f) \cap F(g))\right) \subseteq F(f) \cap F(g)\right)$.

We have

$$
\begin{aligned}
\left\|T_{n} x-T_{n} y\right\| & =\left\|h_{T x}\left(k_{n}\right)-h_{T y}\left(k_{n}\right)\right\| \\
& \leq \Psi\left(k_{n}\right)\|T x-T y\| \quad \text { since } \Gamma \text { is a contractive family } \\
& \leq \Psi\left(k_{n}\right)\{m(x, y)+\operatorname{Ln}(x, y)\} \quad \text { using }(4.1) \\
& =\Psi\left(k_{n}\right) m(x, y)+L_{n} n(x, y)
\end{aligned}
$$

for each $x, y \in F(f) \cap F(g)$, where $L_{n}=L \Psi\left(k_{n}\right), \Psi\left(k_{n}\right) \in(0,1)$,

$$
\begin{aligned}
m(x, y) & =\max \left\{\|f x-g y\|, \operatorname{dist}\left(f x, Y_{q}^{T x}\right), \operatorname{dist}\left(g y, Y_{q}^{T y}\right), \frac{1}{2}\left[\operatorname{dist}\left(g y, Y_{q}^{T x}\right)+\operatorname{dist}\left(f x, Y_{q}^{T y}\right)\right]\right\} \\
& \leq \max \left\{\|f x-g y\|,\left\|f x-T_{n} x\right\|,\left\|g y-T_{n} y\right\|, \frac{1}{2}\left[\left\|g y-T_{n} x\right\|+\left\|f x-T_{n} y\right\|\right]\right\}
\end{aligned}
$$


and

$$
\begin{aligned}
n(x, y) & =\min \left\{\operatorname{dist}\left(f x, Y_{q}^{T x}\right), \operatorname{dist}\left(g y, Y_{q}^{T y}\right), \operatorname{dist}\left(g y, Y_{q}^{T x}\right), \operatorname{dist}\left(f x, Y_{q}^{T y}\right)\right\} \\
& \leq \min \left\{\left\|f x-T_{n} x\right\|,\left\|g y-T_{n} y\right\|,\left\|g y-T_{n} x\right\|,\left\|f x-T_{n} y\right\|\right\} .
\end{aligned}
$$

Thus, for each $n \in \mathbb{N}, T_{n}$ is a generalized almost $(f, g)$-contraction.

If $\operatorname{cl}(T(M))$ is compact, then, for each $n \in \mathbb{N}, \operatorname{cl}\left(T_{n}(M)\right)$ is compact and hence complete. By Theorem 3.5, for each $n \geq 1$, there exists a unique $x_{n} \in F(f) \cap F(g)$ such that $x_{n}=f\left(x_{n}\right)=$ $g\left(x_{n}\right)=T_{n}\left(x_{n}\right)$. Again the compactness of $\operatorname{cl}(T(M))$ implies that there exists a subsequence $\left\{T x_{m}\right\}$ of $\left\{T x_{n}\right\}$ such that $T x_{m} \rightarrow z \in \operatorname{cl}(T(M))$. Since $\left\{T x_{m}\right\}$ is a sequence in $T(F(f) \cap F(g))$ and $\operatorname{cl}(T(F(f) \cap F(g))) \subseteq F(f) \cap F(g)$, we have $z \in F(f) \cap F(g)$. Further, the joint continuity of family $\Gamma$ implies that

$$
x_{m}=T_{m} x_{m}=h_{T x_{m}}\left(k_{m}\right) \rightarrow h_{z}(1)=z \quad \text { as } m \rightarrow \infty .
$$

By the continuity of $T$, we obtain $z=T(z)$. Thus, $M \cap F(T) \cap F(f) \cap F(g) \neq \phi$.

The weak compactness of $\operatorname{wcl}(T(M))$ implies that $\operatorname{wcl}\left(T_{n}(M)\right)$ is weakly compact and hence complete due to completeness of $X$. From Theorem 3.5 for each $n \geq 1$, there exists a unique $x_{n} \in F(f) \cap F(g)$ such that $x_{n}=f\left(x_{n}\right)=g\left(x_{n}\right)=T_{n}\left(x_{n}\right)$. The weak compactness of $\operatorname{wcl}(T(M))$ implies that there is a subsequence $\left\{T x_{m}\right\}$ of $\left\{T x_{n}\right\}$ such that $T x_{m}$ converges weakly to $z \in \operatorname{wcl}(T(M))$ as $m \rightarrow \infty$. Since $\left\{T x_{m}\right\}$ is a sequence in $T(F(f) \cap F(g))$ and $\operatorname{wcl}(T(F(f) \cap F(g))) \subseteq F(f) \cap F(g)$, we have $z \in F(f) \cap F(g)$. By the joint weak continuity of the family we obtain

$$
x_{m}=T_{m} x_{m}=h_{T x_{m}}\left(k_{m}\right) \rightarrow h_{z}(1)=z \quad(\text { weakly) as } m \rightarrow \infty .
$$

Since the weak topology is Hausdorff, by weak continuity of $T$, we have $z=T(z)$. Thus, $M \cap F(T) \cap F(f) \cap F(g) \neq \phi$.

Remark 4.2 By comparing Theorem 2.2(i) of Chandok and Narang [27] with the first case of Theorem 4.1 (when $L=0$ ), their assumptions ' $M$ is complete and has a contractive jointly continuous family $\Gamma$ with $g\left(h_{x}(k)\right)=h_{g x}(k)$ and $f\left(h_{x}(k)\right)=h_{f x}(k)$ for $k \in(0,1)$, $\operatorname{cl}(T(M)) \subseteq f(M) \cap g(M)$, the pairs $(T, f)$ and $(T, g)$ are $C_{q}$-commuting and $f, g$ are continuous on $M$ ' are replaced with ' $M$ is nonempty subset, $F(f) \cap F(g)$ is nonempty and has a contractive jointly continuous family $\Gamma$, and $\operatorname{cl}(T(F(f) \cap F(g))) \subseteq F(f) \cap F(g)$ '.

Corollary 4.3 Let $M$ be a nonempty subset of a normed (respectively, Banach) space $X$ and $T, f$, and $g$ be self-maps of $M$. Suppose $F(f) \cap F(g)$ is q-starshaped, $\operatorname{cl}(T(F(f) \cap F(g))) \subseteq$ $F(f) \cap F(g)$ (respectively, $\operatorname{wcl}(T(F(f) \cap F(g))) \subseteq F(f) \cap F(g)$ ), and there exists a constant $L \geq 0$ such that

$$
\|T x-T y\| \leq m(x, y)+\operatorname{Ln}(x, y)
$$

for all $x, y \in M$, where

$$
\begin{aligned}
m(x, y)= & \max \{\|f x-g y\|, \operatorname{dist}(f x,[q, T x]), \operatorname{dist}(g y,[q, T y]), \\
& \left.\frac{1}{2}[\operatorname{dist}(g y,[q, T x])+\operatorname{dist}(f x,[q, T y])]\right\}
\end{aligned}
$$


and

$$
n(x, y)=\min \{\operatorname{dist}(f x,[q, T x]), \operatorname{dist}(g y,[q, T y]), \operatorname{dist}(g y,[q, T x]), \operatorname{dist}(f x,[q, T y])\} .
$$

Then $M \cap F(T) \cap F(f) \cap F(g) \neq \phi$, provided $\mathrm{cl}(T(M))$ is compact (respectively, $\mathrm{wcl}(T(M)$ ) is weakly compact) and $T$ is continuous (respectively, $T$ is weakly continuous).

Remark 4.4 (1) By comparing Theorem 2.3(i) of Abbas and Ilić [15] with the first case of Corollary 4.3 (when $g=f$ ), their assumptions ' $M$ is $q$-starshaped, $\operatorname{cl}(T(M)) \subseteq f(M)$ ), $f$ and $T$ are weakly compatible on $M$ ' are replaced with ' $F(f)$ is $q$-starshaped, $\operatorname{cl}(T(F(f))) \subseteq F(f)$ '.

(2) By comparing Theorem 2.3(ii) of Abbas and Ilić [15] with the second case of Corollary 4.3 (when $g=f$ ), their assumptions ' $M$ is $q$-starshaped, $\operatorname{cl}(T(M)) \subseteq f(M), f$ and $T$ are weakly compatible on $M, f$ is weakly continuous and $f-T$ is demiclosed at 0 ' are replaced with ' $F(f)$ is $q$-starshaped, $\operatorname{cl}(T(F(f))) \subseteq F(f)$ and $T$ is weakly continuous'.

(3) By comparing Theorem 2.4 of Song [19] with the first case of Corollary 4.3 (when $L=0$ ), their assumptions ' $M$ is $q$-starshaped, $\operatorname{cl}(T(M)) \subseteq f(M) \cap g(M)$, the pairs $(T, f)$ and $(T, g)$ are $C_{q}$-commuting, $f$ and $g$ are $q$-affine and continuous on $M$ ' are replaced with ' $F(f) \cap F(g)$ is $q$-starshaped, $\operatorname{cl}(T(F(f) \cap F(g))) \subseteq F(f) \cap F(g)$ '.

Corollary 4.5 Let $M$ be a nonempty subset of a normed (respectively, Banach) space $X$ and $T, f$, and $g$ be self-maps of $M$. If $M$ has a contractive jointly continuous (respectively, jointly weakly continuous) family $\Gamma=\left\{h_{x}: x \in M\right\}$ such that $g\left(h_{x}(k)\right)=h_{g x}(k)$ and $f\left(h_{x}(k)\right)=h_{f x}(k)$ for all $x \in M, k \in[0,1]$. Suppose $F(f) \cap F(g)$ is nonempty, closed (respectively, weakly closed), $\mathrm{cl}(T(M))$ is compact (respectively, $\mathrm{wcl}(T(M))$ is weakly compact), $T$ is continuous (respectively, weakly continuous), $(T, f)$ and $(T, g)$ are Banach operator pair on $M$ and satisfy $(4.1)$. Then $M \cap F(T) \cap F(f) \cap F(g) \neq \phi$.

Proof For each natural number $n$, define $T_{n}: M \rightarrow M$ by $T_{n}(x)=h_{T x}\left(k_{n}\right)$, for all $x \in M$. Clearly, for each $n \geq 1, T_{n}$ is a self-map on $M$. Since $(T, f)$ is Banach operator pair on $M$, for each $x \in F(f)$, we have $T x \in F(f)$. Consider

$$
f\left(T_{n} x\right)=f\left(h_{T x}\left(k_{n}\right)\right)=h_{f T x}\left(k_{n}\right)=h_{T x}\left(k_{n}\right)=T_{n} x
$$

This implies that $T_{n} x \in F(f)$ for each $x \in F(f)$. Thus for each $n \in \mathbb{N},\left(T_{n}, f\right)$ is a Banach operator pair on $M$. Similarly, for each $n \in \mathbb{N},\left(T_{n}, g\right)$ is a Banach operator on $M$. Now the result follows from Theorem 4.1.

Corollary 4.6 Let $X$ be a normed (respectively, Banach) space and let $T, f$, and $g$ be self-maps of $X$. If $p \in X$ and $D \subseteq C, D_{0}:=D \cap F(f) \cap F(g)$ is nonempty, has a contractive jointly continuous (respectively, jointly weakly continuous) family of functions $\Gamma=\left\{h_{x}\right.$ : $\left.x \in D_{0}\right\}, \operatorname{cl}\left(T\left(D_{0}\right)\right) \subseteq D_{0}$ (respectively, $\left.\operatorname{wcl}\left(T\left(D_{0}\right)\right) \subseteq D_{0}\right), \operatorname{cl}(T(D))$ is compact (respectively, $\operatorname{wcl}(T(D))$ is weakly compact), $T$ is continuous on $D$ (respectively, $T$ is weakly continuous) and (4.1) holds for all $x, y \in D$, then $B_{M}(p) \cap F(T) \cap F(f) \cap F(g) \neq \phi$.

Corollary 4.7 Let $X$ be a normed (respectively, Banach) space and $T, f$, and $g$ be self-maps of $X$. If $p \in X$ and $D \subseteq B_{M}(p), D_{0}:=D \cap F(f) \cap F(g)$ is nonempty, has a contractive jointly continuous (respectively, jointly weakly continuous) family of $\Gamma=\left\{h_{x}: x \in D_{0}\right\}, \operatorname{cl}\left(T\left(D_{0}\right)\right) \subseteq$ 
$D_{0}$ (respectively, $\left.\operatorname{wcl}\left(T\left(D_{0}\right)\right) \subseteq D_{0}\right), \operatorname{cl}(T(D))$ is compact (respectively, $\operatorname{wcl}(T(D))$ is weakly compact), $T$ is continuous on $D$ (respectively, $T$ is weakly continuous) and (4.1) holds for all $x, y \in D$, then $B_{M}(p) \cap F(T) \cap F(f) \cap F(g) \neq \phi$.

Remark 4.8 (1) Theorems 4.1 and 4.2 of Chen and Li [10], Theorems 3 and 4 of Chandok and Narang [24] are particular cases of Corollaries 4.6 and 4.7.

(2) By Proposition 2.2 of Chen and Li [10], it can be concluded that Corollary 4.5 extends and generalizes Corollary 2.1 of Shahzad [28].

Now we present two examples in support of Theorem 4.1 and Theorem 3.5 , respectively.

Example 4.9 Let $X=\mathfrak{R}$ be the set of real numbers with the usual norm and $M=[0,1]$. Assume $T(x)=\frac{1}{2}$, for every $x$ in $M$ and define $f, g: M \rightarrow M$ by

$$
f(x)=\left\{\begin{array}{ll}
x, & x \text { is rational, } \\
1-x, & x \text { is irrational, }
\end{array} \quad g(x)=x \quad \text { for all } x \in M .\right.
$$

Then $F(f) \cap F(g)=\{x, x$ is rational in $M\}, \operatorname{cl}(T(F(f) \cap F(g)))=\left\{\frac{1}{2}\right\} \subseteq F(f) \cap F(g)$ and $\operatorname{cl}(T(M))=\left\{\frac{1}{2}\right\}$ is compact. Suppose that $\Gamma=\left\{h_{x}: x \in F(f) \cap F(g)\right\}$ is a family of functions from $[0,1]$ into $F(f) \cap F(g)$, defined by

$$
h_{x}(t)= \begin{cases}1, & x \in F(f) \cap F(g), t \in M \backslash F(f) \cap F(g), \\ t^{2} x, & x, t \in F(f) \cap F(g) .\end{cases}
$$

We observe that the family $\Gamma$ is contractive jointly continuous for $\varphi(t)=t^{2}, t \in(0,1)$. Thus all the conditions of Theorem 4.1 are satisfied. Consequently $T, f$, and $g$ have a common fixed point. Here it is seen that $x=\frac{1}{2}$ is the common fixed point of $T, f$, and $g$.

Remark 4.10 (1) Theorem 2.2(i) of Chandok and Narang [27] cannot apply to Example 4.9 , since $f$ is not continuous.

(2) It is interesting to note that the results of Akbar et al. [22] cannot apply to Example 4.9 , since $F(f) \cap F(g)$ is not $q$-starshaped.

Example 4.11 Let $X=M=\{\alpha, \beta, \gamma, \delta\}$ and let $d: X \times X \rightarrow \mathfrak{R}$ be given as

$$
\begin{array}{ll}
d(\alpha, \beta)=d(\beta, \alpha)=0.5, & d(\alpha, \gamma)=d(\gamma, \alpha)=2.5, \\
d(\alpha, \delta)=d(\delta, \alpha)=1.6, & d(\beta, \gamma)=d(\gamma, \beta)=2.5, \\
d(\beta, \delta)=d(\delta, \beta)=1.5, & d(\gamma, \delta)=d(\delta, \gamma)=2 \quad \text { and } \\
d(\alpha, \alpha)=d(\beta, \beta)=d(\gamma, \gamma)=d(\delta, \delta)=(0,0) .
\end{array}
$$

Then $(X, d)$ is a metric space. Let $T, f, g: M \rightarrow M$ is defined, respectively, as follows:

$$
T(x)= \begin{cases}\beta, & x \neq \gamma \\ \delta, & x=\gamma\end{cases}
$$


and

$$
\begin{array}{llll}
f \alpha=\beta, & f \beta=\beta, & f \gamma=\alpha, & f \delta=\beta, \\
g \alpha=\delta, & g \beta=\beta, & g \gamma=\gamma, & g \delta=\alpha .
\end{array}
$$

Clearly $F(f) \cap F(g)=\{\beta\}$ and $\operatorname{cl}(T(F(f) \cap F(g)))=\{\beta\} \subseteq F(f) \cap F(g)$. Further $T$ is a generalized almost $(f, g)$-contraction for $\delta=\frac{19}{20}$ and $L=0$. Hence, all the conditions of Theorem 3.5 are satisfied. Consequently $T, f$, and $g$ have a unique common fixed point. Here it is seen that $x=\beta$ is the unique common fixed point of $T, f$, and $g$.

Remark 4.12 (1) In Example 4.11, $f(M)=\{\alpha, \beta\}, g(M)=\{\alpha, \beta, \gamma, \delta\}$ and $T(M)=\{\beta, \delta\}$, therefore $\operatorname{cl} T(M)$ is not contained in $f(M) \cap g(M)$. Hence Theorem 2.1 of Song [19] cannot apply to Example 4.11.

(2) In Example 4.11, if we take $g(x)=f(x)=\left\{\begin{array}{ll}\beta, & x \neq \gamma, \\ \alpha, & x=\gamma,\end{array}\right.$, then $T$ and $f$ does not satisfy the contractive condition of Lemma 3.1 of [10] and Theorem 2.2 of [16] at $x=\gamma, y=\alpha$. Hence Lemma 3.1 of [10] and Theorem 2.2 of [16] cannot apply to Example 4.11.

Remark 4.13 (1) Example 3.3 satisfies all the conditions of Theorem 3.5 except the condition $\operatorname{cl}(T(F(f) \cap F(g))) \subseteq F(f) \cap F(g)$. Note that $F(T) \cap F(f) \cap F(g) \neq \phi$.

\section{Competing interests}

The authors declare that they have no competing interests.

\section{Authors' contributions}

Both authors contributed equally and significantly in writing this paper. Both authors read and approved the final manuscript.

\section{Acknowledgements}

The author would like to thank the referees for their valuable suggestions, which helped to improve the presentation of the paper.

Received: 7 July 2013 Accepted: 5 January 2014 Published: 24 Jan 2014

\section{References}

1. Berinde, V: Approximation fixed points of weak contractions using the Picard iteration. Nonlinear Anal. Forum 9(1), 43-53 (2004)

2. Berinde, V: General constructive fixed point theorems for Ćirić-type almost contractions in metric spaces. Carpath J. Math. 24(2), 10-19 (2008)

3. Kannan, R: Some results on fixed points. Bull. Calcutta Math. Soc. 10, 71-76 (1968)

4. Chatterjea, SK: Fixed point theorem. C. R. Acad. Bulgare Sci. 25, 727-730 (1972)

5. Zamfirescu, T: Fix point theorems in metric spaces. Arch. Math. 23, 292-298 (1972)

6. Babu, GVR, Sandhya, ML, Kameswari, MVR: A note on a fixed point theorem of Berinde on weak contractions. Carpath. J. Math. 24(1), 8-12 (2008)

7. Berinde, V: Some remark on a fixed point theorems for Ćirić-type almost contractions. Carpath. J. Math. 25(2), 157-162 (2009)

8. Jungck, G: Compatible mappings and common fixed points. Int. J. Math. Math. Sci. 9(4), 771-779 (1986) doi:10.1155/s0161171286000935

9. Jungck, G, Rhoades, BE: Fixed points for set valued functions without continuity. Indian J. Pure Appl. Math. 29, 227-238 (1998)

10. Chen, J, Li, Z: Common fixed points for Banach operators pairs in best approximation. J. Math. Anal. Appl. 336 1466-1475 (2007)

11. Al-Thagafi, MA, Shahzad, N: Noncommuting selfmaps and invariant approximations. Nonlinear Anal. 64, 2778-2786 (2006)

12. Shahzad, N: Noncommuting maps and best approximations. Rad. Mat. 10(1), 77-83 (2000/01)

13. Shahzad, N: Invariant approximation and R-sub-weakly commuting maps. J. Math. Anal. Appl. 257, $39-45$ (2001)

14. Naimpally, SA, Singh, KL, Whitfield, JHM: Fixed points and nonexpansive retracts in locally convex spaces. Fundam. Math. 120, 63-75 (1984)

15. Abbas, M, Ilić, D: Common fixed points generalized almost nonexpansive mappings. Filomat 24, 11-18 (2010) 
16. Al-Thagafi, MA, Shahzad, N: Banach operator pairs, common fixed points, invariant approximations, and *-nonexpansive multimaps. Nonlinear Anal. 69, 2733-2739 (2008)

17. Shahzad, N: Invariant approximations, generalized /-contractions, and R-sub-weakly commuting maps. Fixed Point Theory Appl. 1, 79-86 (2005)

18. Hussain, N, Jungck, G: Common fixed point and invariant approximation results for noncommuting generalized ( $f, g)$-nonexpansive maps. J. Math. Anal. Appl. 2, 851-861 (2006)

19. Song, Y: Common fixed points and invariant approximations for generalized $(f, g)$-nonexpansive mappings. Commun. Math. Anal. 2,17-26 (2007)

20. Singh, SP, Watson, B, Srivastava, P: Fixed Point Theory and Best Approximation: The KKM-Map Principle. Kluwer Academic, Dordrecht (1997)

21. Hussain, N, Cho, YJ: Weak contractions, common fixed points, and invariant approximations. J. Inequal. Appl. 2009, Article ID 390634 (2009)

22. Akbar, F, Khan, AR, Sultana, N: Common fixed point and approximation results for generalized $(f, g)$-weak contraction. Fixed Point Theory Appl. 2012, 75 (2012)

23. Dotson, WJ Jr.: On fixed points of nonexpansive mappings in non-convex sets. Proc. Am. Math. Soc. 38, 155-156 (1973)

24. Chandok, S, Narang, TD: Some common fixed point theorems for Banach operator pairs with applications in best approximation. Nonlinear Anal. 73, 105-109 (2010)

25. Al-Thagafi, MA: Common fixed points and best approximation. J. Approx. Theory 85, 318-323 (1996). doi:10.1006/jath.1996.0045

26. Jungck, G, Sessa, S: Fixed point theorems in best approximation theory. Math. Jpn. 42, 249-252 (1995)

27. Chandok, S, Narang, TD: Common fixed points and invariant approximations for $C_{q}$-commuting generalized nonexpansive mappings. Iran. J. Math. Sci. Inform. 7, 21-34 (2012)

28. Shahzad, N: On R-sub-weakly commuting maps and invariant approximations in Banach spaces. Georgian Math. J. $12,157-162(2005)$

29. O'Regan, D, Shahzad, N: Invariant approximations for generalized /-contractions. Numer. Funct. Anal. Optim. 26 565-575 (2005)

30. Shahzad, N, Bassindowa, G: Fixed point theorems for Suzuki-generalized nonexpansive mappings with applications. J. Nonlinear Convex Anal. 13(4), 657-666 (2012)

$10.1186 / 1687-1812-2014-23$

Cite this article as: Rathee and Kumar: Some common fixed-point and invariant approximation results with

generalized almost contractions. Fixed Point Theory and Applications 2014, 2014:23

\section{Submit your manuscript to a SpringerOpen ${ }^{\circ}$ journal and benefit from:}

- Convenient online submission

- Rigorous peer review

- Immediate publication on acceptance

- Open access: articles freely available online

- High visibility within the field

- Retaining the copyright to your article 